\title{
Ecophysiological and Biochemical Changes in Abelmoschus esculentus (L.) Moench under Chromium stress
}

\author{
P. Unnikannan*, P. Vedhanarayanan ${ }^{* *}$, P. Sundaramoorthy*** \\ Department of Botany, Annamalai University, Annamalainagar - 608 002, Tamilnadu, India \\ ***Fax: +91-41-44-222265 \\ ${ }^{*-* * * E-m a i l ~ a d d r e s s: ~ u n e e e k a @ g m a i l . c o m ~, ~ v e d h a a p @ g m a i l . c o m ~, p p s m o o r t h y @ y a h o o . c o m ~}$
}

\begin{abstract}
Increasing concentrations of chromium caused reduction in growth, chlorophyll contents and mineral mutrients of bhendhi. At increasing concentrations of chromium, all the attributes were found to be reduced. Chromium is one of the toxic elements discharging from tannery industry. The long disposal of tannery effluent to the agricultural land causes serious damages in the soil texture; reduce the growth of microorganisms and agricultural crops. Large areas of Vellore district of Tamilnadu was seriously affected by the tannery effluent irrigation to the agricultural land. The present study was conducted to identify the phytotoxic effect and physiological changes of Abelmoschus esculentus (L.) Moench under the treatment of different concentrations of chromium solutions $(2.5,5,10,25,50,75$ and $100 \mathrm{mg} / \mathrm{l})$. The result shows that there was a gradual reduction in the morphological parameters (root length, shoot length, fresh weight and dry weight). Similarly the photosynthetic pigments (Chlorophyll and carotenoid) and biochemical parameters such as protein, amino acid, and sugars were also gradually decreased with increasing concentrations of chromium.
\end{abstract}

Keywords: Abelmoschus esculentus; chromium; biochemical parameters

\section{INTRODUCTION}

Water is the most precious thing for all living organisms in the world. Nowadays, most of our water resources are gradually becoming polluted by various human activities. Water pollution may be defined as a natural or induced change in the quality of water which renders it unusable or dangerous as regard to food, human and animal health, industry, agriculture and fishing (Manna and Das, 2004). The addition of huge amount of sewage, industrial and agricultural effluents is the main cause for water pollution. The effluent discharging industries are tanneries, textile mills, sugar mills, chemical industries, electroplating, distilleries, pharmaceuticals, dairy etc., cause the water and soil pollution. Among the effluent discharging industries, tannery industry plays a major role in polluting the environment. It is one of the oldest cottage industries of India, which stands third largest producer of leather in the world and earn a lot of country's economy (Dadhich et al., 2002)

The major pollutants are sodium chloride, sodium sulphate, lime, chromium, proteinaceous matter and so on (Gomathi et al., 2004). Tannery effluent also contains large 
amounts of other pollutants such as protein, hair, salt, lime, sludge and acid. Among them, chromium is a main constituent of the tannery effluent. Chromium merits a special reference for its toxic potential. In addition, it is released from electroplating, textile printing and metal finishing industries (Sankar Ganesh et al., 2006). Chromium exists in two forms: Hexavalent chromium and trivalent chromium. Hexavalent $\mathrm{Cr}$ is more toxic than trivalent $\mathrm{Cr}$ because of its soluble nature in water. The long-term disposal of tannery wastes onto land can indeed lead to accumulation and transport of water (Sethunathan et al., 2005). Even lower concentration of chromium $(>2 \mathrm{ppm})$ has also been reported to be inhibitory for plant growth. In addition, its presence in excess amount within the plant caused stunted growth, roots develop poorly and leaves appear discolored (Nath et al., 2005). The present study was aimed to find out the phytotoxic effect and physiological changes of Abelmoschus esculentus grown under different concentrations of chromium solution.

\section{MATERIALS AND METHODS}

The experimental crop bhendi (Abelmoschus esculentus (L.) Moench.) was collected from Authorized Private Agro Centre, Chidambaram, Cuddalore district, Tamil Nadu.

A known weight $(5.912 \mathrm{~g})$ of potassium dichromate $\left(\mathrm{K}_{2} \mathrm{Cr}_{2} \mathrm{O}_{7}\right)$ salt was dissolved in $1000 \mathrm{ml}$ of distilled water. This is equal to $1000 \mathrm{ml} / 1$ of chromium. From this stock solution, different concentrations $(2.5,5.0,10.0,25.0,50.0 \mathrm{mg} / \mathrm{l})$ of chromium solution were prepared and they were used for both the experiments.

Pot culture experiments were conducted in Botanical garden, Department of Botany, Annamalai University. The pots were filled with $5 \mathrm{~kg}$ of garden soil. Then, ten seeds of bhendi were sown in each pot. The pots were irrigated with equal volume of different concentrations of chromium solution $(2.5,5,10,25,50,75$ and $100 \mathrm{mg} / 1)$. The control set was maintained with borewell water. After one week, thinning was done by allow to grow 5 seedlings per pot. Five plants were randomly selected from each treatment for the observation of the morphological growth parameters. The shoot length, root length, number of leaves, total leaf area, fresh weight and dry weight were recorded at every 25 days viz., 25, 50 and 75 DAS. The yield parameters were observed and recorded at the time of harvest.

The photosynthetic pigments such as chlorophylls and carotenoid contents were measured by using the standard procedure mentioned by Arnon (1949) and Kirk and Allen (1965) respectively. Simultaneously the biochemical parameters such as Amino acid (Moore and Stein, 1948), Protein (Lowry et al., 1951), reducing, sugar Non-reducing sugar and total sugar (Nelson, 1944) were also calculated at 25, 50 and 75 DAS.

\section{1. Soil Analyses}

The soil samples were collected from each pot and labelled separately after harvesting of Bhendi. The physico-chemical properties of, chromium polluted soil. The various soil properties such as $\mathrm{pH}$, electrical conductivity, nitrogen (Subbiah and Asija, 1976), phosphorus (Jackson, 1958), potassium (Jackson, 1958), copper, zinc, iron, manganese and chromium (Piper, 1966) were analysed and reported. 


\section{RESULT AND DISCUSSION}

\section{1. Shoot length and root length (cm/plant)}

The effect of different concentrations of chromium on shoot length and root length of bhendi crop at various stages of its growth is given in Table 1. The highest root length (8.36, $15.5,17.5 \mathrm{~cm} /$ plant $)$, shoot length $(26.0,37.5,58.5 \mathrm{~cm} /$ plant $)$ were observed at $(25,50,75$ DAS) in control. The minimum range of root length $(3.40,9.20,11.20 \mathrm{~cm} /$ plant $)$ and shoot length $(9.64,21.5,38.5 \mathrm{~cm} /$ plant $)$ were observed at $(25,50,75 \mathrm{DAS})$ in $50 \mathrm{mg} / 1$ concentration of chromium treated bhendi crop. The reduction in plant height might be mainly due to the reduced root growth and consequent lesser nutrients and water transport to the above parts of the plant. In addition to this $\mathrm{Cr}$ transport to the aerial part of the plant have a direct impact on the shoot metabolism contributing to the reduction in plant height (Shankar et al., 2005).

Table 1. Effect of various concentrations of chromium on root length and shoot length $(\mathrm{cm} / \mathrm{plant})$ of A. esculentus at various stages of its growth.

\begin{tabular}{|c|c|c|c|c|c|c|}
\hline \multirow{2}{*}{$\begin{array}{c}\text { Chromium } \\
\text { concentration } \\
(\mathrm{mg} / \mathrm{l})\end{array}$} & \multicolumn{3}{|c|}{ Root length } & \multicolumn{3}{|c|}{ Shoot length } \\
\hline & 25 DAS & 50 DAS & 75 DAS & 25 DAS & 50 DAS & 75 DAS \\
\hline Control & $\begin{array}{c}8.36 \\
\pm 0.418\end{array}$ & $\begin{array}{c}15.5 \\
\pm 0.775\end{array}$ & $\begin{array}{c}17.5 \\
\pm 0.875\end{array}$ & $\begin{array}{c}26.0 \\
\pm 1.300\end{array}$ & $\begin{array}{c}37.5 \\
\pm 1.875\end{array}$ & $\begin{array}{c}58.5 \\
\pm 2.925\end{array}$ \\
\hline 2.5 & $\begin{array}{c}8.10 \\
\pm 0.405\end{array}$ & $\begin{array}{c}13.2 \\
\pm 0.660\end{array}$ & $\begin{array}{c}16.2 \\
\pm 0.810\end{array}$ & $\begin{array}{c}19.20 \\
\pm 0.960\end{array}$ & $\begin{array}{c}36.2 \\
\pm 1.810\end{array}$ & $\begin{array}{c}57.0 \\
\pm 2.850\end{array}$ \\
\hline 5 & $\begin{array}{c}6.40 \\
\pm 0.320\end{array}$ & $\begin{array}{c}13.0 \\
\pm 0.650\end{array}$ & $\begin{array}{c}14.5 \\
\pm 0.725\end{array}$ & $\begin{array}{c}17.70 \\
\pm 0.885\end{array}$ & $\begin{array}{c}32.0 \\
\pm 1.600\end{array}$ & $\begin{array}{c}53.2 \\
\pm 2.660\end{array}$ \\
\hline 10 & $\begin{array}{c}5.96 \\
\pm 0.298\end{array}$ & $\begin{array}{c}12.5 \\
\pm 0.625\end{array}$ & $\begin{array}{c}13.00 \\
\pm 0.650\end{array}$ & $\begin{array}{c}16.00 \\
\pm 0.800\end{array}$ & $\begin{array}{c}28.5 \\
\pm 1.425\end{array}$ & $\begin{array}{c}50.1 \\
\pm 2.660\end{array}$ \\
\hline 25 & $\begin{array}{c}4.80 \\
\pm 0.240\end{array}$ & $\begin{array}{c}10.8 \\
\pm 0.540\end{array}$ & $\begin{array}{c}12.30 \\
\pm 0.615\end{array}$ & $\begin{array}{c}12.44 \\
\pm 0.622\end{array}$ & $\begin{array}{c}23.2 \\
\pm 1.160\end{array}$ & $\begin{array}{c}47.3 \\
\pm 2.365\end{array}$ \\
\hline 50 & $\begin{array}{c}3.40 \\
\pm 0.170\end{array}$ & $\begin{aligned} & 9.2 \\
\pm & 0.460\end{aligned}$ & $\begin{array}{c}11.20 \\
\pm 0.560\end{array}$ & $\begin{array}{c}9.64 \\
\pm 0.482\end{array}$ & $\begin{array}{c}21.5 \\
\pm 1.075\end{array}$ & $\begin{array}{c}38.5 \\
\pm 1.925\end{array}$ \\
\hline
\end{tabular}




\section{2. Number of leaves and total leaf area}

The effect of different concentrations of chromium on number of leaves and total leaf area of bhendi crop at various stages of its growth is shown in Table 2. The higher amount of leaves $(5.0,6.0,7.0$ leaves/plant) and the lower number of leaves (3.0, 4.0, 5.0 leaves/plant) were observed in control at $25^{\text {th }}, 50^{\text {th }}, 75^{\text {th }}$ days respectively. The maximum leaf area $(66.0$, $138.6,255.74 \mathrm{~cm}^{2} /$ plant $)$ and the minimum leaf area $\left(18.37,27.72,79.5 \mathrm{~cm}^{2} /\right.$ plant $)$ were observed in $50 \mathrm{mg} / \mathrm{l}$ concentration of chromium treated plant at 25, 50, 75 DAS respectively. The reduced cell size and decreased intracellular spaces were largely responsible for reduction in leaf area. The size of the areoles decreased in plants treated with chromium and this may be due to the decrease in leaf area (Joshi et al., 1999).

Table 2. Effect of various concentrations of chromium on number of leaves and total leaf area $\left(\mathrm{cm}^{2} /\right.$ plant $)$ of $A$. esculentus at various stages of its growth.

\begin{tabular}{|c|c|c|c|c|c|c|}
\hline \multirow{2}{*}{$\begin{array}{c}\text { Chromium } \\
\text { concentration } \\
(\mathbf{m g} / \mathbf{l})\end{array}$} & \multicolumn{3}{|c|}{ Number of leaves } & \multicolumn{3}{|c|}{ Total leaf area } \\
\hline & 25 DAS & 50 DAS & 75 DAS & 25 DAS & 50 DAS & 75 DAS \\
\hline Control & $\begin{array}{c}5.0 \\
\pm 0.250\end{array}$ & $\begin{array}{c}6.0 \\
\pm 0.300\end{array}$ & $\begin{array}{c}7.0 \\
\pm 0.350\end{array}$ & $\begin{array}{c}66.0 \\
\pm 3.300\end{array}$ & $\begin{array}{c}138.6 \\
\pm 6.930\end{array}$ & $\begin{array}{r}255.74 \\
\pm 12.787\end{array}$ \\
\hline 2.5 & $\begin{aligned} & 5.0 \\
\pm & 0.250\end{aligned}$ & $\begin{array}{c}6.0 \\
\pm 0.300\end{array}$ & $\begin{array}{c}6.0 \\
\pm 0.300\end{array}$ & $\begin{array}{c}57.75 \\
\pm 2.887\end{array}$ & $\begin{array}{c}95.04 \\
\pm 4.752\end{array}$ & $\begin{array}{r}178.20 \\
\pm 8.910\end{array}$ \\
\hline 5 & $\begin{array}{c}4.0 \\
\pm 0.200\end{array}$ & $\begin{array}{c}5.0 \\
\pm 0.250\end{array}$ & $\begin{array}{c}6.0 \\
\pm 0.300\end{array}$ & $\begin{array}{c}36.96 \\
\pm 1.848\end{array}$ & $\begin{array}{c}57.75 \\
\pm 2.887\end{array}$ & $\begin{array}{r}158.40 \\
\pm 7.920\end{array}$ \\
\hline 10 & $\begin{array}{c}4.0 \\
\pm 0.200\end{array}$ & $\begin{array}{c}5.0 \\
\pm 0.250\end{array}$ & $\begin{array}{c}6.0 \\
\pm 0.300\end{array}$ & $\begin{array}{c}32.34 \\
\pm 1.617\end{array}$ & $\begin{array}{c}49.5 \\
\pm 2.475\end{array}$ & $\begin{array}{r}126.72 \\
\pm 6.336\end{array}$ \\
\hline 25 & $\begin{aligned} & 3.0 \\
\pm & 0.150\end{aligned}$ & $\begin{array}{c}4.0 \\
\pm 0.200\end{array}$ & $\begin{aligned} & 6.0 \\
\pm & 0.300\end{aligned}$ & $\begin{array}{c}20.79 \\
\pm 1.039\end{array}$ & $\begin{array}{c}36.96 \\
\pm 1.848\end{array}$ & $\begin{array}{r}110.88 \\
\pm 5.544\end{array}$ \\
\hline 50 & $\begin{array}{l}3.0 \\
\pm 0.150\end{array}$ & $\begin{aligned} & 4.0 \\
\pm & 0.200\end{aligned}$ & $\begin{aligned} & 5.0 \\
\pm & 0.250\end{aligned}$ & $\begin{array}{c}18.37 \\
\pm 0.918\end{array}$ & $\begin{array}{c}27.72 \\
\pm 1.386\end{array}$ & $\begin{array}{c}79.5 \\
+3.975\end{array}$ \\
\hline
\end{tabular}




\section{3. Fresh weight and dry weight (g/plant)}

The effect of different concentrations of chromium on fresh weight and dry weight of bhendi crop at various stages of its growth is given in Table 3. The highest amount of fresh weight $(2.152,5.328,9.750 \mathrm{~g} /$ plant $)$ and dry weight $(1.120,2.106,3.235 \mathrm{~g} /$ plant $)$ were observed in control at 25, 50 and 75 DAS. The minimum amount of fresh weight $(0.513$, $2.300,5.323 \mathrm{~g} / \mathrm{plant})$ and dry weight $(0.168,0.885,1.955 \mathrm{~g} / \mathrm{plant})$ were observed in $50 \mathrm{mg} / \mathrm{l}$ concentration of chromium treated plant at $25,50,75$ DAS respectively. The concentration of soluble protein provide an evidence that chromium is responsible for enhanced degradative pathways as it occurs in tissues during cellular breakdown of the constituents.

Table 3. Effect of various concentrations of chromium on fresh weight and dry weight (g/plant) of $A$. esculentus at various stages of its growth.

\begin{tabular}{|c|c|c|c|c|c|c|}
\hline \multirow{2}{*}{$\begin{array}{c}\text { Chromium } \\
\text { concentration } \\
(\mathrm{mg} / \mathrm{l})\end{array}$} & \multicolumn{3}{|c|}{ Fresh weight } & \multicolumn{3}{|c|}{ Dry weight } \\
\hline & 25 DAS & 50 DAS & 75 DAS & 25 DAS & 50 DAS & 75 DAS \\
\hline Control & $\begin{array}{c}2.152 \\
\pm 0.1076\end{array}$ & $\begin{array}{c}5.328 \\
\pm 0.2664\end{array}$ & $\begin{array}{c}9.750 \\
\pm 0.4875\end{array}$ & $\begin{array}{c}1.120 \\
\pm 0.056\end{array}$ & $\begin{array}{c}2.106 \\
\pm 0.1053\end{array}$ & $\begin{array}{r}3.235 \\
\pm 0.1617\end{array}$ \\
\hline 2.5 & $\begin{array}{c}1.856 \\
\pm 0.0928\end{array}$ & $\begin{array}{c}4.742 \\
\pm 0.2371\end{array}$ & $\begin{array}{c}9.380 \\
\pm 0.469\end{array}$ & $\begin{array}{c}0.832 \\
\pm 0.0416\end{array}$ & $\begin{array}{c}2.060 \\
\pm 0.103\end{array}$ & $\begin{array}{c}3.162 \\
\pm 0.1581\end{array}$ \\
\hline 5 & $\begin{array}{c}1.630 \\
\pm 0.0815\end{array}$ & $\begin{array}{c}4.311 \\
\pm 0.2155\end{array}$ & $\begin{array}{c}8.112 \\
\pm 0.4056\end{array}$ & $\begin{array}{c}0.513 \\
\pm 0.0256\end{array}$ & $\begin{array}{c}1.723 \\
\pm 0.0861\end{array}$ & $\begin{array}{c}2.478 \\
\pm 0.1239\end{array}$ \\
\hline 10 & $\begin{array}{c}1.516 \\
\pm 0.0758\end{array}$ & $\begin{array}{c}3.260 \\
\pm 0.163\end{array}$ & $\begin{array}{c}7.355 \\
\pm 0.3677\end{array}$ & $\begin{array}{c}0.420 \\
\pm 0.021\end{array}$ & $\begin{array}{c}1.620 \\
\pm 0.0810\end{array}$ & $\begin{array}{c}2.315 \\
\pm 0.1157\end{array}$ \\
\hline 25 & $\begin{array}{c}0.865 \\
\pm 0.0432\end{array}$ & $\begin{array}{c}3.175 \\
\pm 0.3677\end{array}$ & $\begin{array}{c}6.412 \\
\pm 0.3206\end{array}$ & $\begin{array}{c}0.283 \\
\pm 0.0141\end{array}$ & $\begin{array}{c}1.123 \\
\pm 0.0561\end{array}$ & $\begin{array}{c}2.100 \\
\pm 0.1050\end{array}$ \\
\hline 50 & $\begin{array}{c}0.513 \\
\pm 0.0256\end{array}$ & $\begin{array}{c}2.300 \\
\pm 0.115\end{array}$ & $\begin{array}{c}5.323 \\
\pm 0.2661\end{array}$ & $\begin{array}{c}0.168 \\
\pm 0.0084\end{array}$ & $\begin{array}{c}0.885 \\
\pm 0.0442\end{array}$ & $\begin{array}{c}1.955 \\
\pm 0.977\end{array}$ \\
\hline
\end{tabular}

\section{4. Yield parameters}

The effect of different concentrations of chromium on yield parameters of bhendi crop is given in Table 4 . The maximum number of fruits $(15.0 /$ plant $)$ fruit length $(13.5 \mathrm{~cm} /$ fruit $)$, 
fruit weight (3.25 g/fruit), number of seeds (45.5/fruit) and 100 seed weight $(8.88 \mathrm{~g})$ were noted in control. The minimum amount of fruit number $(6.0 /$ plant $)$, fruit length $(6.0 \mathrm{~cm} /$ fruit $)$, fruit weight (1.00 g/fruit), number of seeds (18.0/fruit) and 100 seeds weight $(2.15 \mathrm{~g})$ were observed in 50 per cent of chromium concentrated bhendi crop. $\mathrm{Cr}$ on plant processes during early growth and development culminates in reduction of yield and total dry matter as a consequence of poor production, translocation and partitioning of assimilates to the economic parts of the plant (Sharma and Sharma, 1993).

Table 4. Effect of different concentrations of chromium on yield parameters of A. esculentus.

\begin{tabular}{cccccc}
\hline $\begin{array}{c}\text { Chromium } \\
\text { concentration } \\
(\mathbf{m g} / \mathbf{l})\end{array}$ & $\begin{array}{c}\text { Number of } \\
\text { fruits per } \\
\text { plant }\end{array}$ & $\begin{array}{c}\text { Fruit length } \\
\text { (cm/fruit) }\end{array}$ & $\begin{array}{c}\text { Fruit weight } \\
\text { (g/fruit) }\end{array}$ & $\begin{array}{c}\text { Number of } \\
\text { seeds per } \\
\text { fruit }\end{array}$ & $\begin{array}{c}\text { 100 seed } \\
\text { weight (g) }\end{array}$ \\
\hline Control & 15.0 & 13.5 & 3.250 & 45.5 & 8.880 \\
& \pm 0.75 & \pm 0.67 & \pm 0.1625 & \pm 2.275 & \pm 0.444 \\
2.5 & 14.0 & 12.2 & 3.100 & 40.2 & 7.680 \\
& \pm 0.70 & \pm 0.61 & \pm 0.1550 & \pm 2.010 & \pm 0.384 \\
5 & 12.0 & 10.5 & 2.780 & 35.0 & 6.210 \\
& \pm 0.60 & \pm 0.52 & \pm 0.1390 & \pm 1.750 & \pm 0.310 \\
& 10.0 & 9.6 & 2.150 & 26.2 & 5.000 \\
10 & \pm 0.50 & \pm 0.48 & \pm 0.1075 & \pm 1.310 & \pm 0.250 \\
& 8.0 & 8.2 & 1.520 & 20.5 & 4.320 \\
& \pm 0.40 & \pm 0.41 & \pm 0.0760 & \pm 1.025 & \pm 0.216 \\
& & & & & \\
& 6.0 & 6.0 & 1.000 & 18.0 & 2.150 \\
& \pm 0.30 & \pm 0.30 & \pm 0.0500 & \pm 0.900 & \pm 0.107 \\
\hline
\end{tabular}

\section{5. Photosynthetic pigments}

The effect of various concentrations of chromium on chlorophyll ' $a$ ', chlorophyll ' $b$ ', total chlorophyll and carotenoid content (mg/g fr. wt.) of bhendi crop at $25^{\text {th }}$ and $75^{\text {th }}$ days are shown in Table 5. The higher amount of chlorophyll ' $a$ ', chlorophyll ' $b$ ', total chlorophyll and carotenoid content $(0.4973,0.3921,0.8894$ and $0.6297 \mathrm{mg} / \mathrm{g}$ fr. wt. $)$ were observed at $75^{\text {th }}$ days of control plant. Similarly, the lowest values of chlorophyll ' $a$ ', chlorophyll ' $b$ ', total chlorophyll and carotenoid content $(0.1591,0.0914,0.2533$ and $0.2215 \mathrm{mg} / \mathrm{g}$ fr. wt) were observed at in $50 \mathrm{mg} / \mathrm{l}$ concentration of chromium irrigated bhendi crop. The impaired $\alpha$-amino laevulinic acid dehydratase activity leading to reduced photosynthetic pigments has been observed in chromium treated Nymphaea alba (Vajpyee et al., 2000). Besides, lipid peroxidation also caused the degradation of photosynthetic pigments (Rai et al., 2004). The reduction photosynthetic pigments may also be due to the disruption of chloroplast phosphorylation observed in various plants as suggested by Jana and Choudhari (1992); Chandra and Kulshrentha (2004); Singh and Sinha (2005). 
Table 5. Effect of various concentrations of chromium on photosynthetic pigments (mg/g fr. wt) of $A$. esculentus at various stages of its growth.

25 DAS

\begin{tabular}{|c|c|c|c|c|c|c|c|c|}
\hline $\begin{array}{c}\text { Chromium } \\
\text { concentrations } \\
(\mathbf{m g} / \mathbf{l})\end{array}$ & 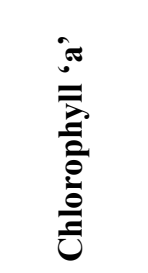 & 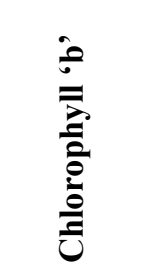 & 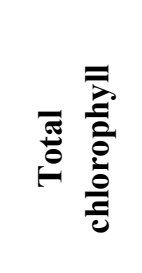 & :등 & 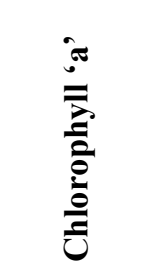 & 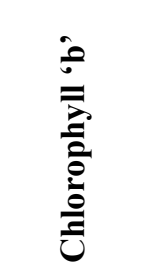 & 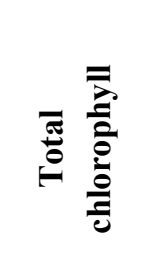 & 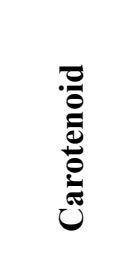 \\
\hline Control & $\begin{array}{c}0.3289 \\
\pm 0.0164\end{array}$ & $\begin{array}{r}0.2359 \\
\pm 0.1179\end{array}$ & $\begin{array}{r}0.5705 \\
\pm 0.0282\end{array}$ & $\begin{array}{c}0.4832 \\
\pm 0.0241\end{array}$ & $\begin{array}{c}0.4973 \\
\pm 0.0248\end{array}$ & $\begin{array}{c}0.3921 \\
\pm 0.0196\end{array}$ & $\begin{array}{c}0.8894 \\
\pm 0.0444\end{array}$ & $\begin{array}{c}0.6297 \\
\pm 0 \\
0314\end{array}$ \\
\hline 2.5 & $\begin{array}{c}0.3185 \\
\pm 0.0159\end{array}$ & $\begin{array}{c}0.2058 \\
\pm 0.0102\end{array}$ & $\begin{array}{c}0.5298 \\
\pm 0.0249\end{array}$ & $\begin{array}{c}0.4316 \\
\pm 0.0215\end{array}$ & $\begin{array}{c}0.4071 \\
\pm 0.0203\end{array}$ & $\begin{array}{c}0.3610 \\
\pm 0.0180\end{array}$ & $\begin{array}{c}0.7375 \\
\pm 0.0368\end{array}$ & $\begin{array}{c}0.6976 \\
\pm 0.0348\end{array}$ \\
\hline 5 & $\begin{array}{c}0.2118 \\
\pm 0.0105\end{array}$ & $\begin{array}{c}0.1014 \\
\pm 0.0050\end{array}$ & $\begin{array}{c}0.3168 \\
\pm 0.0158\end{array}$ & $\begin{array}{c}0.3015 \\
\pm 0.0150\end{array}$ & $\begin{array}{c}0.3975 \\
\pm 0.0198\end{array}$ & $\begin{array}{c}0.2923 \\
\pm 0.0146\end{array}$ & $\begin{array}{c}0.5890 \\
\pm 0.0294\end{array}$ & $\begin{array}{c}0.6520 \\
\pm 0.0326\end{array}$ \\
\hline 10 & $\begin{array}{c}0.1949 \\
\pm 0.0097\end{array}$ & $\begin{array}{c}0.1013 \\
\pm 0.0506\end{array}$ & $\begin{array}{c}0.2996 \\
\pm 0.0149\end{array}$ & $\begin{array}{c}0.2738 \\
\pm 0.0136\end{array}$ & $\begin{array}{c}0.2750 \\
\pm 0.0137\end{array}$ & $\begin{array}{c}0.1924 \\
\pm 0.0096\end{array}$ & $\begin{array}{c}0.3752 \\
\pm 0.0187\end{array}$ & $\begin{array}{c}0.4971 \\
\pm 0.0248\end{array}$ \\
\hline 25 & $\begin{array}{c}0.1932 \\
\pm 0.0096\end{array}$ & $\begin{array}{c}0.0923 \\
\pm 0.0046\end{array}$ & $\begin{array}{c}0.2889 \\
\pm 0.0144\end{array}$ & $\begin{array}{c}0.2338 \\
\pm 0.0116\end{array}$ & $\begin{array}{c}0.2075 \\
\pm 0.0103\end{array}$ & $\begin{array}{c}0.1250 \\
\pm 0.0002\end{array}$ & $\begin{array}{c}0.3074 \\
\pm 0.0153\end{array}$ & $\begin{array}{c}0.3720 \\
\pm 0.0186\end{array}$ \\
\hline 50 & $\begin{array}{c}0.1591 \\
\pm 0.0079\end{array}$ & $\begin{array}{c}0.0914 \\
\pm 0.0045\end{array}$ & $\begin{array}{c}0.2533 \\
\pm 0.0126\end{array}$ & $\begin{array}{c}0.2216 \\
\pm 0.0110\end{array}$ & $\begin{array}{c}0.1694 \\
\pm 0.0084\end{array}$ & $\begin{array}{c}0.1095 \\
\pm 0.0054\end{array}$ & $\begin{array}{c}0.2973 \\
\pm 0.0148\end{array}$ & $\begin{array}{r}0.2590 \\
\pm 0.0129\end{array}$ \\
\hline
\end{tabular}

\section{6. Protein (mg/g fr. wt.)}

The influence of different concentrations of chromium on protein content of bhendi at $25^{\text {th }}$ and $75^{\text {th }}$ day of its growth is given in Table 6.

The maximum protein content of various parts of bhendi (leaf 2.9702, stem 1.0741, root $3.9740 \mathrm{mg} / \mathrm{g}$ fr. wt.) was observed in $25^{\text {th }}$ day of control plants irrigated with tap water.

The minimum protein content (leaf 0.8973 , stem 0.6294 , root $1.2973 \mathrm{mg} / \mathrm{g}$ fr. wt.) was recorded at $75^{\text {th }}$ day of its growth in $50 \mathrm{mg} / \mathrm{l}$ concentration of chromium treatment.

The degradation in protein content might be the result of increased activity of the protease or other catabolic enzymes under chromium stress (Vajpayee et al., 2001; Rai et al., 1992). During transport of heavy metals in plants, sulphydryl group of protein may be reduced causing deleterious effect in the normal protein form. 
Table 6. Effect of different concentrations of chromium on protein content (mg/g fr. wt) of $A$ esculentus at two stages of its growth.

\begin{tabular}{|c|c|c|c|c|c|c|}
\hline \multirow{2}{*}{$\begin{array}{c}\text { Chromium } \\
\text { concentrations } \\
(\mathbf{m g} / \mathbf{l})\end{array}$} & \multicolumn{3}{|c|}{25} & \multicolumn{3}{|c|}{75} \\
\hline & Leaf & Stem & Root & Leaf & Stem & Root \\
\hline Control & $\begin{array}{c}2.9702 \\
\pm 0.1485\end{array}$ & $\begin{array}{c}1.0741 \\
\pm 0.0537\end{array}$ & $\begin{array}{c}3.9740 \\
\pm 0.1987\end{array}$ & $\begin{array}{c}3.2175 \\
\pm 0.1608\end{array}$ & $\begin{array}{c}1.3890 \\
\pm 0.0694\end{array}$ & $\begin{array}{c}4.1273 \\
\pm 0.2063\end{array}$ \\
\hline 2.5 & $\begin{array}{c}2.7301 \\
\pm 0.1365\end{array}$ & $\begin{array}{c}0.9736 \\
\pm 0.0486\end{array}$ & $\begin{array}{c}3.6607 \\
\pm 0.1830\end{array}$ & $\begin{array}{c}2.9381 \\
\pm 0.1469\end{array}$ & $\begin{array}{c}1.2940 \\
\pm 0.0647\end{array}$ & $\begin{array}{r}3.7940 \\
\pm 0.1897\end{array}$ \\
\hline 5 & $\begin{array}{r}1.7940 \\
\pm 0.0897\end{array}$ & $\begin{array}{r}0.9372 \\
\pm 0.0468\end{array}$ & $\begin{array}{c}3.2940 \\
\pm 0.1647\end{array}$ & $\begin{array}{c}2.5701 \\
\pm 0.1285\end{array}$ & $\begin{array}{r}1.0794 \\
\pm 0.0539\end{array}$ & $\begin{array}{c}2.9731 \\
\pm 0.1486\end{array}$ \\
\hline 10 & $\begin{array}{c}1.0761 \\
\pm 0.0538\end{array}$ & $\begin{array}{c}0.8713 \\
\pm 0.0435\end{array}$ & $\begin{array}{c}2.1720 \\
\pm 0.1086\end{array}$ & $\begin{array}{c}1.9047 \\
\pm 0.0952\end{array}$ & $\begin{array}{c}0.9764 \\
\pm 0.0488\end{array}$ & $\begin{array}{c}2.7690 \\
\pm 0.1384\end{array}$ \\
\hline 25 & $\begin{array}{c}0.9786 \\
\pm 0.0489\end{array}$ & $\begin{array}{c}0.8601 \\
\pm 0.0430\end{array}$ & $\begin{array}{c}2.0621 \\
\pm 0.1031\end{array}$ & $\begin{array}{c}1.2714 \\
\pm 0.0635\end{array}$ & $\begin{array}{c}0.8731 \\
\pm 0.0436\end{array}$ & $\begin{array}{c}2.9902 \\
\pm 0.1495\end{array}$ \\
\hline 50 & $\begin{array}{c}0.6317 \\
\pm 0.3150\end{array}$ & $\begin{array}{c}0.5250 \\
\pm 0.0262\end{array}$ & $\begin{array}{c}1.0731 \\
\pm 0.0536\end{array}$ & $\begin{array}{c}0.8973 \\
\pm 0.0048\end{array}$ & $\begin{array}{c}0.6294 \\
\pm 0.0314\end{array}$ & $\begin{array}{c}1.2973 \\
\pm 0.0648\end{array}$ \\
\hline
\end{tabular}

\section{7. Amino acid (mg/g fr. wt.)}

The effect of various concentrations of chromium on amino acid content ( $\mathrm{mg} / \mathrm{g}$ fr. wt.) of bhendi at $25^{\text {th }}$ and $75^{\text {th }}$ day of its growth is given in Table 7.

The maximum amount of amino acid content (leaf 4.7804, stem 1.7674 , root 3.9701 $\mathrm{mg} / \mathrm{g}$ fr. wt) was observed in $75^{\text {th }}$ days of control plants.

The minimum amount of amino acid content (leaf 1.8740 , stem 0.5470 , root $1.4076 \mathrm{mg} / \mathrm{g}$ fr. wt) was recorded in $25^{\text {th }}$ day of its growth at $50 \mathrm{mg} / \mathrm{l}$ chromium concentration.

The decline in amino acid cysteine may result in the degradation of sulphate reducing enzymes leading to toxic effects (Vajpayee et al., 2001). 
Table 7. Effect of different concentrations of chromium on amino acid content ( $\mathrm{mg} / \mathrm{g}$ fr. wt) of bhendi (A. esculentus) at two stages of its growth.

\begin{tabular}{|c|c|c|c|c|c|c|}
\hline \multirow{2}{*}{$\begin{array}{c}\text { Chromium } \\
\text { concentrations } \\
(\mathbf{m g} / \mathbf{l})\end{array}$} & \multicolumn{3}{|c|}{25 DAS } & \multicolumn{3}{|c|}{75 DAS } \\
\hline & Leaf & Stem & Root & Leaf & Stem & Root \\
\hline Control & $\begin{array}{c}4.224 \\
\pm 0.2112\end{array}$ & $\begin{array}{r}1.2902 \\
\pm 0.0645\end{array}$ & $\begin{array}{c}3.8970 \\
\pm 0.1948\end{array}$ & $\begin{array}{c}4.7804 \\
\pm 0.2390\end{array}$ & $\begin{array}{c}1.7674 \\
\pm 0.0883\end{array}$ & $\begin{array}{r}3.9701 \\
\pm 0.1985\end{array}$ \\
\hline 2.5 & $\begin{array}{c}3.682 \\
\pm 0.1841\end{array}$ & $\begin{array}{c}1.2902 \\
\pm 0.0645\end{array}$ & $\begin{array}{c}3.2731 \\
\pm 0.1636\end{array}$ & $\begin{array}{c}3.9709 \\
\pm 0.1985\end{array}$ & $\begin{array}{c}1.3844 \\
\pm 0.0692\end{array}$ & $\begin{array}{r}3.487 \\
\pm 0.1743\end{array}$ \\
\hline 5 & $\begin{array}{c}3.524 \\
+0.1762\end{array}$ & $\begin{array}{c}1.0840 \\
\pm 0.0542\end{array}$ & $\begin{array}{c}3.0740 \\
\pm 0.1537\end{array}$ & $\begin{array}{c}3.8021 \\
\pm 0.1901\end{array}$ & $\begin{array}{c}1.1707 \\
\pm 0.0585\end{array}$ & $\begin{array}{r}3.2670 \\
\pm 0.1633\end{array}$ \\
\hline 10 & $\begin{array}{c}2.996 \\
\pm 0.1498\end{array}$ & $\begin{array}{c}0.6180 \\
\pm 0.0309\end{array}$ & $\begin{array}{c}2.4570 \\
\pm 0.1228\end{array}$ & $\begin{array}{c}3.6974 \\
\pm 0.1848\end{array}$ & $\begin{array}{c}0.9671 \\
\pm 0.0483\end{array}$ & $\begin{array}{r}2.9741 \\
\pm 0.1487\end{array}$ \\
\hline 25 & $\begin{array}{c}2.587 \\
\pm 0.1293\end{array}$ & $\begin{array}{c}0.7621 \\
\pm 0.0381\end{array}$ & $\begin{array}{r}1.9670 \\
\pm 0.0983\end{array}$ & $\begin{array}{c}2.2840 \\
\pm 0.1142\end{array}$ & $\begin{array}{c}0.8812 \\
\pm 0.0440\end{array}$ & $\begin{array}{r}2.3174 \\
\pm 0.1158\end{array}$ \\
\hline 50 & $\begin{array}{c}1.874 \\
\pm 0.0937\end{array}$ & $\begin{array}{c}0.5470 \\
\pm 0.0273\end{array}$ & $\begin{array}{c}1.4076 \\
\pm 0.0703\end{array}$ & $\begin{array}{c}2.0701 \\
\pm 0.1035\end{array}$ & $\begin{array}{c}0.7381 \\
\pm 0.0369\end{array}$ & $\begin{array}{r}1.0793 \\
\pm 0.0539\end{array}$ \\
\hline
\end{tabular}

\section{8. Sugar (mg/g fr. wt.)}

The impact of different concentrations of chromium on reducing sugar content $(\mathrm{mg} / \mathrm{g} \mathrm{fr}$. wt.) in various parts of bhendi in $25^{\text {th }}$ and $75^{\text {th }}$ day is given in Table 8 . The highest amount of reducing sugar of leaf (2.6640), stem (1.5000), root (1.4120 mg/g fr. wt.) was observed in control plant at $75^{\text {th }}$ day. The lowest content of reducing sugar of leaf $(1.4940 \mathrm{mg} / \mathrm{g} \mathrm{fr}$. wt.), stem $\left(0.5160 \mathrm{mg} / \mathrm{g}\right.$ fr. wt.), root $(0.5000 \mathrm{mg} / \mathrm{g}$ fr. wt. $)$ were recorded at $25^{\text {th }}$ days of growth in 50 $\mathrm{mg} / \mathrm{l}$ concentration of chromium irrigation. The maximum non-reducing sugar content of leaf $\left(7.146 \mathrm{mg} / \mathrm{g}\right.$ fr. wt.), stem $(6.215 \mathrm{mg} / \mathrm{g}$ fr. wt. $)$, and $\operatorname{root}\left(5.000 \mathrm{mg} \mathrm{g}^{-1} \mathrm{fr}\right.$. wt.) were recorded at $75^{\text {th }}$ days of control plant. The lowest amount of non-reducing sugar in leaf $(1.598 \mathrm{mg} / \mathrm{g} \mathrm{fr}$. wt.), stem $(0.726 \mathrm{mg} / \mathrm{g}$ fr. wt. $)$, root $\left(0.785 \mathrm{mg} \mathrm{g}^{-1} \mathrm{fr}\right.$. wt. $)$ were noted at $25^{\text {th }}$ days of its growth in 50 $\mathrm{mg} / \mathrm{l}$ concentration of chromium irrigation (Table 9). The higher amount of total sugar content in various parts like (leaf 13.258, stem 10.877, root $9.102 \mathrm{mg} / \mathrm{g}$ fr. wt.) were noted in $75^{\text {th }}$ days of control plant. 
Table 8. Effect of different concentrations of chromium on reducing sugar content (mg/g fr. wt) of $A$. esculentus at two stages of its growth.

\begin{tabular}{|c|c|c|c|c|c|c|}
\hline \multirow{2}{*}{$\begin{array}{c}\text { Chromium } \\
\text { concentrations } \\
(\mathrm{mg} / \mathrm{l})\end{array}$} & \multicolumn{3}{|c|}{25 DAS } & \multicolumn{3}{|c|}{75 DAS } \\
\hline & Leaf & Stem & Root & Leaf & Stem & Root \\
\hline Control & $\begin{array}{c}2.664 \\
\pm 0.1332\end{array}$ & $\begin{array}{c}1.500 \\
\pm 0.075\end{array}$ & $\begin{array}{c}1.412 \\
\pm 0.0706\end{array}$ & $\begin{array}{c}6.112 \\
\pm 0.3056\end{array}$ & $\begin{array}{c}4.662 \\
\pm 0.2331\end{array}$ & $\begin{array}{c}4.102 \\
\pm 0.2051\end{array}$ \\
\hline 2.5 & $\begin{array}{c}2.382 \\
\pm 0.1191\end{array}$ & $\begin{array}{c}1.326 \\
\pm 0.0663\end{array}$ & $\begin{array}{c}1.212 \\
\pm 0.0606\end{array}$ & $\begin{array}{c}6.015 \\
\pm 0.3007\end{array}$ & $\begin{array}{c}4.106 \\
\pm 0.2053\end{array}$ & $\begin{array}{c}3.737 \\
\pm 0.1868\end{array}$ \\
\hline 5 & $\begin{array}{c}2.175 \\
\pm 0.1087\end{array}$ & $\begin{array}{c}1.110 \\
\pm 0.0555\end{array}$ & $\begin{array}{c}1.011 \\
\pm 0.0505\end{array}$ & $\begin{array}{c}5.328 \\
\pm 0.2664\end{array}$ & $\begin{array}{c}3.715 \\
\pm 0.1857\end{array}$ & $\begin{array}{c}3.400 \\
\pm 0.1700\end{array}$ \\
\hline 10 & $\begin{array}{c}2.072 \\
\pm 0.1036\end{array}$ & $\begin{array}{c}0.865 \\
\pm 0.0432\end{array}$ & $\begin{array}{c}0.735 \\
\pm 0.0367\end{array}$ & $\begin{array}{c}4.672 \\
\pm 0.2336\end{array}$ & $\begin{array}{c}2.635 \\
\pm 0.1317\end{array}$ & $\begin{aligned} & 2.550 \\
\pm & 0.1275\end{aligned}$ \\
\hline 25 & $\begin{array}{c}1.953 \\
\pm 0.0976\end{array}$ & $\begin{array}{c}0.732 \\
\pm 0.0366\end{array}$ & $\begin{array}{c}0.660 \\
\pm 0.0330\end{array}$ & $\begin{array}{c}4.155 \\
\pm 0.2077\end{array}$ & $\begin{array}{c}2.143 \\
\pm 0.1071\end{array}$ & $\begin{array}{c}2.000 \\
\pm 0.1000\end{array}$ \\
\hline 50 & $\begin{array}{c}1.494 \\
\pm 0.0747\end{array}$ & $\begin{array}{c}0.516 \\
\pm 0.0258\end{array}$ & $\begin{array}{c}0.500 \\
\pm 0.0250\end{array}$ & $\begin{array}{c}3.556 \\
\pm 0.1778\end{array}$ & $\begin{array}{c}2.106 \\
\pm 0.1053\end{array}$ & $\begin{array}{c}1.916 \\
\pm 0.0956\end{array}$ \\
\hline
\end{tabular}

Table 9. Effect of different concentrations of chromium on non-reducing sugar content ( $\mathrm{mg} / \mathrm{g}$ fr. wt) of $A$. esculentus at two stages of its growth.

\begin{tabular}{ccccccc}
\hline $\begin{array}{c}\text { Chromium } \\
\text { concentrations } \\
(\mathbf{m g} / \mathbf{l})\end{array}$ & Leaf & Stem & Root & Leaf & Stem & Root \\
& & & & & & \\
& & & & & & \\
& & & & & & \\
Control & 2.841 & 2.015 & 1.965 & 7.146 & 6.215 & 5.000 \\
& \pm 0.1420 & \pm 0.1007 & \pm 0.0982 & \pm 0.3573 & \pm 0.3107 & \pm 0.2500 \\
\hline
\end{tabular}




\begin{tabular}{ccccccc}
\hline 2.5 & 2.678 & 1.812 & 1.732 & 6.785 & 5.880 & 4.810 \\
& \pm 0.1339 & \pm 0.0906 & \pm 0.0866 & \pm 0.3392 & \pm 0.2940 & \pm 0.2405 \\
& & & & & & \\
5 & 2.442 & 1.432 & 1.106 & 5.886 & 5.410 & 4.312 \\
& \pm 0.1121 & \pm 0.0716 & \pm 0.0553 & \pm 0.2943 & \pm 0.2705 & \pm 0.2156 \\
& & & & & & \\
10 & 2.220 & 0.916 & 0.985 & 5.102 & 4.762 & 3.615 \\
& \pm 0.1110 & \pm 0.0458 & \pm 0.0492 & \pm 0.2551 & \pm 0.2381 & \pm 0.1807 \\
& & & & & & \\
& 2.072 & 0.815 & 0.876 & 4.660 & 4.312 & 3.288 \\
& \pm 0.1036 & \pm 0.0407 & \pm 0.0438 & \pm 0.2330 & \pm 0.2156 & \pm 0.1644 \\
& & & & & & \\
& 1.598 & 0.726 & 0.785 & 4.215 & 3.816 & 2.432 \\
& \pm 0.0799 & \pm 0.0362 & \pm 0.0392 & \pm 0.2107 & \pm 0.1908 & \pm 0.1216 \\
& & & & & & \\
\hline
\end{tabular}

Table 10. Effect of different concentrations of chromium on total sugar content (mg/g fr. wt) of $A$. esculentus at two stages of its growth.

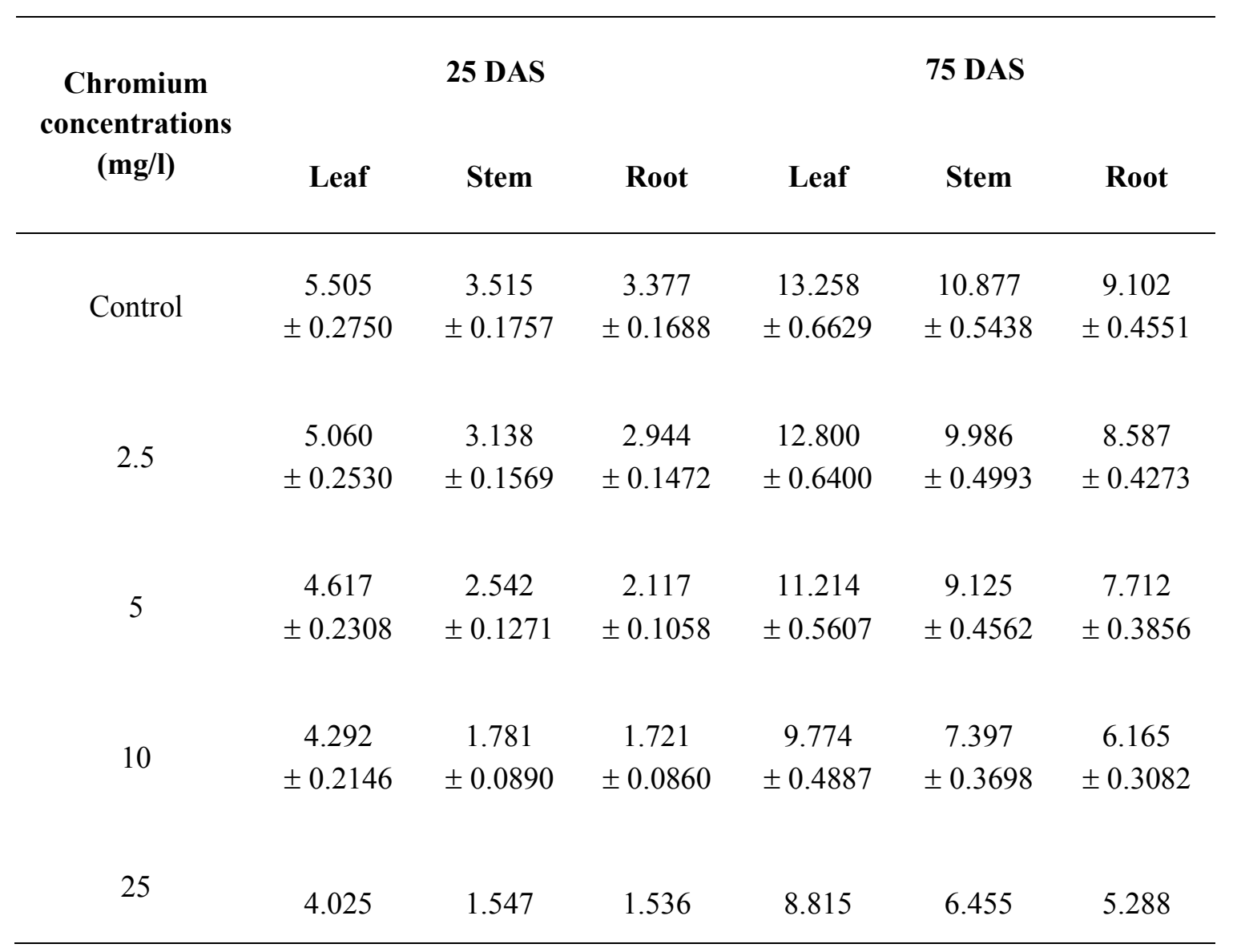




\begin{tabular}{ccccccc}
\hline & \pm 0.2012 & \pm 0.0773 & \pm 0.7680 & \pm 0.4407 & \pm 0.3227 & \pm 0.2644 \\
& & & & & & \\
50 & 3.092 & 1.242 & 1.285 & 7.771 & 5.922 & 4.348 \\
& \pm 0.1546 & \pm 0.0621 & \pm 0.0642 & \pm 0.3885 & \pm 0.2961 & \pm 0.2174
\end{tabular}

The lowest total sugar content (leaf 3.092, stem 1.242, root $1.285 \mathrm{mg} / \mathrm{g}$ fr. wt.) were observed at $75^{\text {th }}$ days of its growth in $50 \mathrm{mg} / \mathrm{l}$ concentration of chromium irrigation (Table 10). Loss of sugar formation may also be due to the conversion of sugar into energy when the plants were stressed (Outridge and Noler, 1991). It may be also due to the imbalance which might eventually lead to depletion of carbohydrate reserve (Mahadeswaraswamy and Theresa, 1992).

\section{9. Analysis of chromium polluted soil}

Physico-chemical properties of different concentrations of chromium polluted soil are given in Table 11. The bhendi crop grown chromium polluted soil revealed that they are having the values of $\mathrm{pH}(0.18), \mathrm{EC}(7.8)$, nitrogen $(50 \mathrm{~g} / \mathrm{kg})$, phosphorus $(8.0 \mathrm{~g} / \mathrm{kg})$, potassium $(87$ $\mathrm{g} / \mathrm{kg})$, copper $(14.15 \mathrm{ppm})$, zinc $(11.00 \mathrm{ppm})$, iron $(18.0 \mathrm{ppm})$, manganese $(3.00 \mathrm{ppm})$ and chromium $(1107 \mathrm{mg} / \mathrm{g})$ were observed at $50 \mathrm{mg} / 1$ concentration of chromium polluted soil.

The control soil are having $\mathrm{pH}(7.6 \mathrm{ppm}), \mathrm{EC}(0.39 \mathrm{ppm})$, nitrogen $(46 \mathrm{~g} / \mathrm{kg})$, phosphorus (9 $\mathrm{g} / \mathrm{kg})$, potassium $(500 \mathrm{~g} / \mathrm{kg})$, copper (21.15 ppm), zinc (15.20 ppm), iron (22.95 ppm), manganese (5.15 ppm) with nil chromium.

Table 11. Physicochemical analyses of different concentrations of chromium polluted soil.

\begin{tabular}{ccccccccccc}
\hline $\begin{array}{c}\text { Chromium } \\
\text { concentrations } \\
(\mathrm{mg} / \mathrm{l})\end{array}$ & $\mathrm{pH}$ & $\begin{array}{c}\mathrm{EC} \\
(\mathrm{Mm} \\
\text { hos/cm })\end{array}$ & $\begin{array}{c}\mathrm{N} \\
(\mathrm{g} / \mathrm{kg})\end{array}$ & $\begin{array}{c}\mathrm{P} \\
(\mathrm{g} / \mathrm{kg})\end{array}$ & $\begin{array}{c}\mathrm{K} \\
(\mathrm{g} / \mathrm{kg})\end{array}$ & $\begin{array}{c}\mathrm{Cu} \\
(\mathrm{ppm})\end{array}$ & $\begin{array}{c}\mathrm{Zn} \\
(\mathrm{ppm})\end{array}$ & $\begin{array}{c}\mathrm{Fe} \\
(\mathrm{ppm})\end{array}$ & $\begin{array}{c}\mathrm{Mn} \\
(\mathrm{ppm})\end{array}$ & $\begin{array}{c}\mathrm{Cr} \\
(\mathrm{mg} / \mathrm{kg})\end{array}$ \\
\hline Control & 7.6 & 0.39 & 46 & 9 & 500 & 21.15 & 15.20 & 22.95 & 5.15 & - \\
2.5 & 7.7 & 0.32 & 70 & 11.5 & 82 & 20.10 & 13.80 & 20.00 & 5.00 & 57.0 \\
5 & 8.7 & 0.14 & 46 & 8.5 & 100 & 18.00 & 13.05 & 19.15 & 5.00 & 114.0 \\
10 & 7.9 & 0.22 & 59 & 8.5 & 92 & 16.00 & 12.12 & 19.00 & 4.10 & 217.0 \\
25 & 0.19 & 8.4 & 49 & 10 & 92 & 15.30 & 11.10 & 18.25 & 3.85 & 598.0 \\
50 & 0.18 & 7.8 & 50 & 8.0 & 87 & 14.15 & 11.00 & 18.00 & 3.00 & 110.7 \\
\hline
\end{tabular}




\section{CONCLUSION}

Present study concluded that the phytotoxicity effect of hexavalent $\mathrm{Cr}$ severely affected the growth and biomass of $A$. esculentus. The harmful effects of $\mathrm{Cr}$ on plant's growth and development by extensively investigating the physiological and biochemical parameters. It was known that adverse effects of $\mathrm{Cr}$ were due to the negative impacts on physiological and biochemical parametes. High concentrations of $\mathrm{Cr}$ is highly contaminated soil. The study suggest that the chromium is highly toxic to the growth and development of A.esculentus

\section{References}

[1] Arnon D. I., Plant Physiol. 24 (1949) 1-5.

[2] Chandra P., K. Kulshrestha, Bot. Rev. 70(30) (2004) 313-327.

[3] Dadhich A., Y. A. Maruthi, P. Srinivas, J. Ecotoxicol. Environ. Monit. 12(2) (2002) 139-145.

[4] Gomathi M., A. Subhash, R. Parvatham, J. Ind. Poll. Con. 20(2) (2004) 331-338.

[5] Jackson M. L., Soil chemical analysis, prentice, Hall of India Private Limited, New Delhi, 1958, pp. 22-31.

[6] Jana S., M. A. Choudhuri, New Phytol. 90 (1992) 477-484.

[7] Joshi U. N., S. S. Rathore, S. K. Arora, IJEP 19 (1999) 745-749.

[8] Kirk J. T. O., R. L. Allen, Biochem. Biophys. Res. Cann. 27 (1965) 523-530.

[9] Lowry O. H., N. J. Rosenbrough, A. L. Farr, R. J. Randall, J. Biol. Chem. 193 (1951) 265-275.

[10] Mahadeswaraswamy and Theresa, Geobios 19(6) (1992) 242-246.

[11] Manna R., A.K. Das, Poll. Res. 23(1) (2004) 117-120.

[12] Moore S., W. H. Stein, J. Biol. Chem. 176 (1948) 367-388.

[13] Nath K., S. Saini, Y. K. Sharma, J. Environ. Biol. 26(2) (2005) 197-204.

[14] Nelson N., Anal. Chem. 3 (1944) 426-428.

[15] Outridge P. M., B. N. Noller, Rev. Environ. Contam. Toxicol. 121 (1991) 2-55.

[16] Piper C., Soil and plant analysis. Asian Hans Publishers, Bombay, 1966, p.11-36.

[17] Rai P., S. N. Vajpayee, S. Mehrotra, Plant Sci. 167 (2004) 1154-1169.

[18] Rai V. N., R. D. Tripathi, Kumar, 1992. Chemosphere 25 (1992) 1722-1732.

[19] Sankar Ganesh K., P. Sundaramoorthy, A. L. A. Chidambaram, Poll. Res. 25(4) (2006) 757-761.

[20] Sethunathan N., M. Megharaj, L. Smith, S. P. B. Kamaludeen, S. Avudainayagam, R. Naidu, Agric. Ecosyst. Environment 105 (2005) 657-661.

[21] Sharma D. C., C. P. Sharma, Indian J. Exp. Biol. 34 (1996) 689-691.

[22] Singh S., S. Sinha, Ecotoxicol. Environ. Safety 62(1) (2005) 118-127. 
[23] Subbiah B. V., G. L. Asija, Curr. Sci. (1976) 259-260.

[24] Vajpayee P., R. D. Tripati, U. N. Rai, M. B. Ali, S. N. Singh, Chemosphere 41 (2000) 1075-1082.

[25] Vajpayee P., U. N. Rai, M. B. Ali, R. D. Tripati, U. Yadav, S. Sinha and S. N. Singh, Bull. Environ. Cont. Toxicol. 67 (2001) 246-256. 